\title{
Fewer adults add salt at the table after initiation of a national salt campaign in the UK: a repeated cross-sectional analysis
}

\author{
Jennifer Sutherland $^{1 *}$, Phil Edwards ${ }^{1}$, Bhavani Shankar ${ }^{2}$ and Alan D. Dangour ${ }^{1}$ \\ ${ }^{1}$ London School of Hygiene and Tropical Medicine, Department of Population Health, Keppel Street, \\ London WC1E 7HT, UK \\ ${ }^{2}$ School of Oriental and African Studies and Leverhulme Centre for Integrative Research in Agriculture and Health, \\ 36 Gordon Square, London WC1H OPD, UK \\ (Submitted 15 August 2012 - Final revision received 2 November 2012 - Accepted 2 November 2012 - First published online 3 January 2013)
}

\section{Abstract}

In 2003, the UK Food Standards Agency and the Department of Health began attempts to reduce national salt intakes via reformulation of processed foods and a consumer awareness campaign on the negative impacts of salt on health. The present study uses large nationally representative samples of households in England to assess whether discretionary salt use was affected by the national salt reduction campaign. Large cross-sectional datasets from the Health Survey for England were used to analyse trends in adults adding salt at the table between 1997 and 2007. Since 1997, there has been a steady decline in salt use at the table. Ordinal logistic regression analysis controlling for age, sex, total household income, region, ethnicity and background trends revealed that the reduction in salt use was significantly greater after the campaign (OR 0.58; $95 \%$ CI 0.54, 0.63). Women (OR 0.71; 95\% CI 0.68, 0.74), non-white ethnic groups (OR 0.69; $95 \%$ CI $0.62,0.77$ ), high-income households (OR 0.75; $95 \%$ CI 0.69, 0.82), middle-income households (OR $0.79 ; 95 \%$ CI 0.75 , 0.84 ) and households in central (OR 0.90; $95 \%$ CI 0.84, 0.98) or the south of England (OR 0.82; 95\% CI 0.77, 0.88) were less likely to add salt at the table. The results extend previous evidence of a beneficial response to the salt campaign by demonstrating the effect on salt use at the table. Future programmatic and research efforts may benefit from targeting specific population groups and improving the evidence base for evaluating the impact of the campaign.

Key words: Food Standards Agency: Salt campaign: Salt: Discretionary salt use

Hypertension is recognised as a major cause of CVD, the leading contributor to the global burden of disease ${ }^{(1)}$. Substantial mechanistic and epidemiological evidence links dietary salt intake to hypertension, and the $\mathrm{WHO}^{(1)}$ has recommended reducing salt $(\mathrm{Na})$ intake in whole populations through costeffective public health interventions. In the UK, the Committee on Medical Aspects of Food and Nutrition Policy and latterly the Scientific Advisory Committee on Nutrition have advised that average salt intakes should be reduced to no more than $6 \mathrm{~g} / \mathrm{d}^{(2,3)}$. In 2003, The UK Food Standards Agency (FSA) and the Department of Health in England committed to reducing salt intakes to this level and proposed that this could be attained via two general strategies. First, by a gradual reduction in the salt content of foods through engagement with the food industry on reformulation of industrially processed foods and, second, by increasing consumer awareness of the impact of salt on health via a public awareness campaign using a variety of media outlets to provide consumers with strategies to reduce their salt intake ${ }^{(3)}$.
The UK salt reduction strategy has been hailed as a success in various quarters $^{(4)}$. The FSA's own analyses based on $24 \mathrm{~h}$ urinary $\mathrm{Na}$ excretion levels have suggested that the mean population salt intake, which was as high as $9.5 \mathrm{~g} / \mathrm{d}$ in 2001 , fell after the salt reduction campaign to $9 \mathrm{~g} / \mathrm{d}$ by $2005 / 6^{(5,6)}$, to $8.6 \mathrm{~g} / \mathrm{d}$ by $2008^{(7)}$, and to latest estimates of $8.1 \mathrm{~g} / \mathrm{d}$ in $2011^{(8)}$. Independent econometric analysis of spot urinary Na levels taken between 2003 and 2007 supports a reduction in salt intake of the order of $10 \%$, attributable to the campaign ${ }^{(9)}$. Furthermore, evaluations of consumer behaviour collected in national surveys have revealed increases in both public awareness of the salt campaign and the selection of processed food purchases based on their reported salt content ${ }^{(10,11)}$. Strong demographic and sex differences in these trends have been noted, with lower socio-economic groups and males demonstrating less awareness of the link between salt and health ${ }^{(12)}$.

Previous evaluations of the national salt reduction campaign have tended to focus on overall salt intake, which can conflate the effect of the awareness campaign with the effects flowing

Abbreviations: FSA, Food Standards Agency; HSE, Health Survey for England.

*Corresponding author: J. Sutherland, fax + 44207958 8111, email jennifer.sutherland@lshtm.ac.uk 
from food reformulation, or on public awareness of the campaign. No previous study has assessed directly the effect of the campaign on the addition of salt at the table by consumers. Although the majority of salt intake is from processed foods, salt added at the table or during cooking remains a significant source, with an estimated $15-20 \%$ of salt in the diet obtained from discretionary sources ${ }^{(3)}$. This study uses large nationally representative samples of households in England to assess whether discretionary use of salt at the table was affected by the national salt reduction campaign.

\section{Subjects and methods}

\section{Study design and participants}

Data were obtained from repeated cross-sections of the Health Survey for England (HSE), an annual survey of a nationally representative sample of the general population living in households in England. The HSE utilises a multistage stratified probability sampling design with postcode sectors as the primary sampling unit and the Postcode Address File as the sampling frame for households ${ }^{(13-18)}$. For the purpose of the present study, participants were adults over the age of 16 years in the general population sample of the HSE. Children under the age of 16 years were omitted from the analysis due to inconsistencies in the data on salt use. Ethical approval for each annual data collection cycle of the HSE was obtained from the London Multi-Centre Research Ethics Committee and from all Local Research Ethics Committees in England.

\section{Variables and coding}

The HSE has collected data on reported salt use in the general population in the years 1997, 1998, 2003, 2005, 2006 and 2007. Specifically, participants were asked to categorise their salt use at the table into one of four options: (1) 'generally add salt to food without tasting it first', (2) 'taste the food, but then generally add salt', (3) 'taste the food, but only occasionally add salt', or (4) 'rarely, or never, add salt at the table'. In the primary analysis, salt use was coded as an ordered categorical variable with four levels of discretionary salt use. In further sensitivity analysis, salt use was coded as a binary variable combining the first two options into those who 'generally add salt at the table' and the second two options as those who 'do not generally add salt at the table'.

The HSE questionnaires also provided information on demographic variables. Independent variables were selected and categorised as age (16-24, 25-64 or $65+$ years), ethnic group (white or other), total annual income (low $<£ 23400$, medium $£ 23400-£ 59999$ or high $£ 60000+$ ) and region (north, midlands or south) using data on Government Office Regions. Independent variables were selected where evidence exists showing associations with salt consumption or dietary behaviour $^{(9,12)}$.

\section{Statistical analysis}

The HSE datasets were obtained from the Economic and Social Data Service. Statistical analysis was conducted in
Stata (version 11.0; Stata Corporation) using the 'svy' survey commands to allow for the complex sample design of the HSE. Sex-specific estimates of the percentage of adults generally adding salt were calculated and stratified by independent variables. Multivariable ordinal logistic regression was performed to assess whether the introduction of the salt campaign was associated with salt behaviour at the table, exploiting the ordered ranking to the salt behaviour variable. The salt campaign was included in the model as a binary variable with the years up to and including 2003 as pre-campaign, and the years $2005-7$ as post-campaign. The model also included four socio-economic and demographic variables (age, ethnicity, income and region). Dummy variables for each year were also incorporated to control for possible unknown year-specific shocks or background trends that may have influenced salt use due to factors not otherwise captured in the model. As a sensitivity analysis, logistic regression was also performed, treating responses to the salt behaviour variable as binary. Effects were estimated as OR with $95 \%$ CI.

\section{Weighting}

Weights were used in estimates to account for the HSE sampling design throughout the analysis where possible. As the HSE does not provide probability weights for years before 2003, all statistical analyses were conducted twice: once using all years between 1997 and 2007 but without incorporating weights, and once using the years 2003-7 only and incorporating weights. No weighting for selection bias was required as all adults in responding households were selected ${ }^{(19)}$. When pooling datasets for all years, the individual weights were rescaled to allow for the fact that multiple survey samples were being combined. For this, the individual weights reported for each year were scaled by dividing them by the mean weight for that year, and then in order to account for unequal sample sizes used each year, the rescaled weights were multiplied by the proportion that each annual survey represented of the combined pooled sample ${ }^{(20)}$.

\section{Results}

In total, 67980 individuals answered the HSE salt behaviour question between 1997 and 2007. There was a decrease in the survey response rate from $71 \%$ in 1997 to $58 \%$ in 2007 , and the samples included in the more recent HSE surveys had greater mean age and were more likely to come from higherincome groups than those in the earlier surveys (Table 1).

The proportion of adults who generally added salt at the table (unweighted estimates) decreased from 40.1\% in 1997 to $31.7 \%$ in 2007. This decline was apparent in both men and women, though a consistently lower percentage of women than men reported generally adding salt at the table across all years (Fig. 1). The trend was also present in the weighted estimates for the years 2003-7, which showed a decline in the percentage of adults who generally added salt at the table from $32.5 \%$ in 2003 to $23.2 \%$ in 2007 (Table 2). The decline in the percentage of adults adding salt at the table between 2003 and 2007 was present in both sexes and across all age and income groups, 
Table 1. Characteristics of the participants aged 16 years and over from 1997 to 2007 in the Health Survey for England

\begin{tabular}{|c|c|c|c|c|c|c|}
\hline & 1997 & 1998 & 2003 & 2005 & 2006 & 2007 \\
\hline Sample $(n)$ & 8582 & 15908 & 14836 & 7630 & 14142 & 6882 \\
\hline Sex (\% male) & 45.4 & $45 \cdot 2$ & 44.5 & $45 \cdot 3$ & 44.9 & 44.6 \\
\hline \multicolumn{7}{|l|}{ Age (years) } \\
\hline Mean & $46 \cdot 3$ & $46 \cdot 9$ & $48 \cdot 2$ & $48 \cdot 0$ & $49 \cdot 3$ & 49.1 \\
\hline SD & $18 \cdot 3$ & $18 \cdot 6$ & 18.5 & $18 \cdot 4$ & $18 \cdot 6$ & $18 \cdot 6$ \\
\hline \multicolumn{7}{|l|}{ Region (\%) } \\
\hline North & $25 \cdot 8$ & $30 \cdot 7$ & $29 \cdot 8$ & $48 \cdot 0$ & 46.6 & $45 \cdot 2$ \\
\hline Midlands & $22 \cdot 7$ & $19 \cdot 5$ & $20 \cdot 3$ & 39.5 & $40 \cdot 3$ & $40 \cdot 4$ \\
\hline South & $51 \cdot 6$ & $49 \cdot 8$ & $49 \cdot 9$ & 12.5 & $13 \cdot 1$ & 14.3 \\
\hline \multicolumn{7}{|l|}{ Income (\%) } \\
\hline Low & 64.9 & $62 \cdot 9$ & $51 \cdot 6$ & 48.0 & 46.6 & $45 \cdot 0$ \\
\hline Medium & $30 \cdot 8$ & $32 \cdot 0$ & 38.5 & 39.4 & $40 \cdot 3$ & 40.5 \\
\hline High & 4.4 & $5 \cdot 1$ & 9.9 & $12 \cdot 6$ & $13 \cdot 1$ & 14.6 \\
\hline \multicolumn{7}{|l|}{ Ethnic group (\%) } \\
\hline White & 94.3 & $94 \cdot 1$ & $92 \cdot 1$ & $92 \cdot 0$ & 90.7 & 89.6 \\
\hline Other & $5 \cdot 7$ & 5.9 & $7 \cdot 9$ & 8.0 & 9.3 & $10 \cdot 4$ \\
\hline Response rate (\%) & 71 & 69 & 66 & 64 & 61 & 58 \\
\hline
\end{tabular}

as well as in all regions and ethnic groups. However, differences in the proportions of adults adding salt at the table between subgroups were apparent. Compared with younger age groups, a greater proportion of adults aged over 65 years reported that they generally added salt at the table in all years except 2003. Compared with adults in the highest-income group, adults in the low-income group were consistently more likely to add salt at the table across all years. Adding salt at the table was most common in adults living in the north and least common in adults living in the south of England across all years. Finally, compared with other ethnic groups, white populations were more likely to add salt at the table across all survey years.

After adjusting for age, sex, region, total annual income, ethnicity and year-specific shocks, ordinal logistic regression analysis revealed that the odds of adding salt at the table were significantly lower after the introduction of the national salt reduction campaign (OR 0.58; 95\% CI 0.54, 0.63; Table 3). In the adjusted model, women were less likely to add salt at the table than men (OR 0.71; $95 \%$ CI $0.68,0.74$ ); adults living in central England (OR 0.90; 95\% CI 0.84, 0.98) and southern England (OR 0.82; $95 \%$ CI 0.77, 0.88) were less likely to add salt at the table compared with adults living in northern England. The total annual household income was significantly associated with salt use at the table, with those in the higher-income group (OR 0.75; 95\% CI $0.69,0.82$ ) and medium-income group (OR 0.79; $95 \% \mathrm{CI}$ $0.75,0.84)$ being less likely to add salt at the table compared with those in the low-income group. Adults in non-white ethnic groups were also less likely to add salt at the table compared with adults in the majority white ethnic group

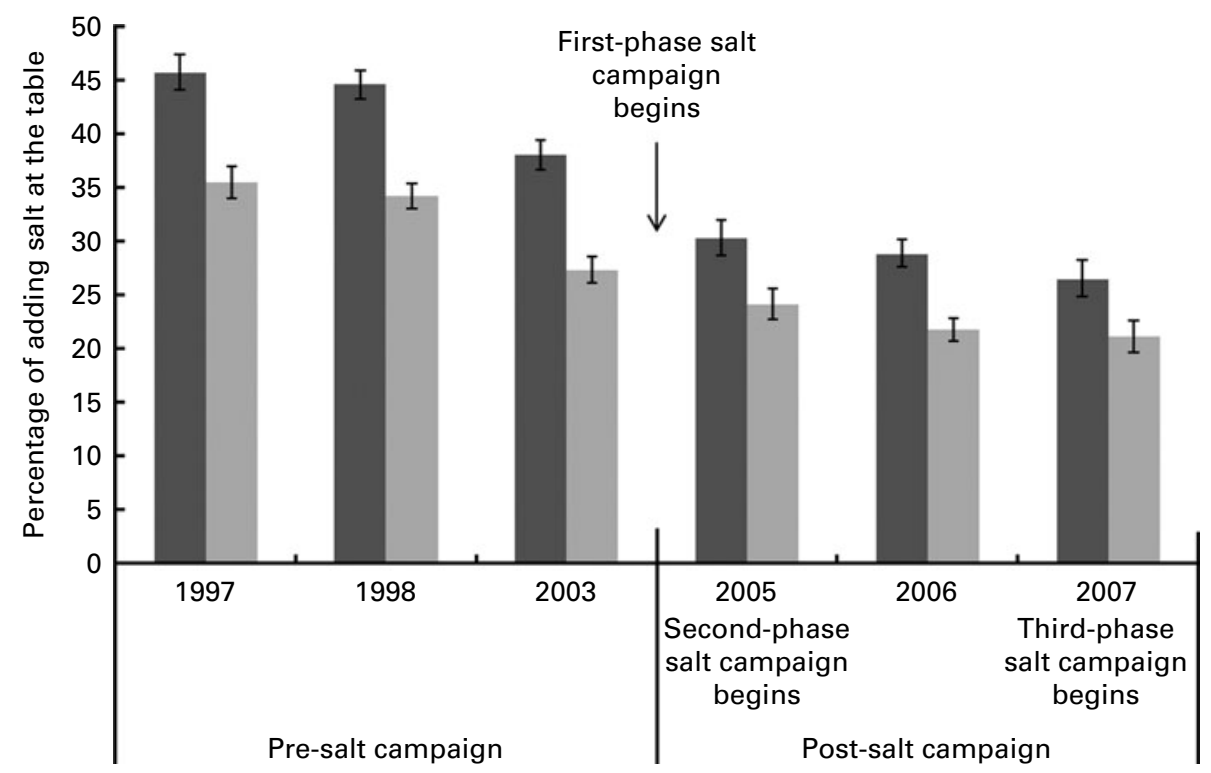

Fig. 1. Proportion of men $(\square)$ and women $(\square)$ over 16 years who 'generally add salt at the table', 1997 to 2007 . Values are percentages, with confidence intervals represented by vertical bars. Estimates shown are an weighted in order to display maximum years of the Health Survey for England data. 
Table 2. Percentage of adults who 'generally add salt at the table' by age, sex, region and income, from 2003 to 2007 (weighted estimates) (Percentages and $95 \%$ confidence intervals)

\begin{tabular}{|c|c|c|c|c|c|c|c|c|}
\hline & \multicolumn{2}{|c|}{2003} & \multicolumn{2}{|c|}{2005} & \multicolumn{2}{|c|}{2006} & \multicolumn{2}{|c|}{2007} \\
\hline & $\%$ & $95 \% \mathrm{Cl}$ & $\%$ & $95 \% \mathrm{Cl}$ & $\%$ & $95 \% \mathrm{Cl}$ & $\%$ & $95 \% \mathrm{Cl}$ \\
\hline All & $32 \cdot 5$ & $31 \cdot 4,33 \cdot 6$ & $27 \cdot 0$ & $25 \cdot 7,28 \cdot 3$ & $24 \cdot 8$ & $23 \cdot 8,25 \cdot 9$ & $23 \cdot 2$ & $21 \cdot 9,24 \cdot 6$ \\
\hline \multicolumn{9}{|l|}{ Sex } \\
\hline Men & $37 \cdot 7$ & $36 \cdot 3,39 \cdot 2$ & $30 \cdot 1$ & $28 \cdot 4,31.9$ & $28 \cdot 1$ & $26 \cdot 7,29 \cdot 5$ & $25 \cdot 6$ & $23 \cdot 9,27 \cdot 5$ \\
\hline Women & $27 \cdot 5$ & $26 \cdot 2,28 \cdot 8$ & $24 \cdot 0$ & $22 \cdot 6,25 \cdot 6$ & $21 \cdot 8$ & $20 \cdot 7,22 \cdot 8$ & $20 \cdot 9$ & $19 \cdot 4,22 \cdot 4$ \\
\hline \multicolumn{9}{|l|}{ Age (years) } \\
\hline $16-24$ & $37 \cdot 4$ & $34.3,40.5$ & $27 \cdot 9$ & $24 \cdot 7,31 \cdot 3$ & $23 \cdot 0$ & $20 \cdot 6,25 \cdot 6$ & $23 \cdot 0$ & $19 \cdot 6,26 \cdot 7$ \\
\hline $25-64$ & 31.7 & $30 \cdot 5,33 \cdot 0$ & $26 \cdot 4$ & $25 \cdot 0,27 \cdot 8$ & $25 \cdot 0$ & $23 \cdot 8,26 \cdot 2$ & $22 \cdot 4$ & $20 \cdot 9,24 \cdot 0$ \\
\hline $65+$ & $32 \cdot 1$ & $30 \cdot 2,34 \cdot 0$ & 28.5 & $26 \cdot 2,30 \cdot 9$ & $25 \cdot 7$ & $24 \cdot 2,27 \cdot 4$ & $26 \cdot 3$ & $23 \cdot 9,28 \cdot 8$ \\
\hline \multicolumn{9}{|l|}{ Total income } \\
\hline Low & $34 \cdot 8$ & $33 \cdot 2,36 \cdot 4$ & $30 \cdot 1$ & $28 \cdot 2,32 \cdot 1$ & $27 \cdot 1$ & $25 \cdot 7,28 \cdot 6$ & $27 \cdot 3$ & $25 \cdot 0,29 \cdot 7$ \\
\hline Medium & $30 \cdot 4$ & $28 \cdot 6,32 \cdot 2$ & 24.8 & $22 \cdot 8,26 \cdot 9$ & 22.5 & $21 \cdot 1,24 \cdot 0$ & 21.9 & $19 \cdot 8,24 \cdot 0$ \\
\hline High & $29 \cdot 1$ & $25 \cdot 8,32 \cdot 6$ & $19 \cdot 1$ & $16 \cdot 1,22 \cdot 5$ & $22 \cdot 2$ & $19 \cdot 5,25 \cdot 1$ & $17 \cdot 4$ & $14 \cdot 3,20 \cdot 9$ \\
\hline \multicolumn{9}{|l|}{ Region } \\
\hline North & $37 \cdot 1$ & $35 \cdot 2,39 \cdot 1$ & $28 \cdot 8$ & $26 \cdot 6,31 \cdot 1$ & $27 \cdot 4$ & $25 \cdot 6,29 \cdot 3$ & $28 \cdot 7$ & $26 \cdot 2,31 \cdot 4$ \\
\hline Midlands & $31 \cdot 7$ & $29 \cdot 6,34 \cdot 0$ & $26 \cdot 9$ & $24 \cdot 4,29 \cdot 6$ & $26 \cdot 1$ & $24 \cdot 1,28 \cdot 1$ & $21 \cdot 8$ & $19 \cdot 0,24 \cdot 8$ \\
\hline South & 30.0 & $28.5,31.5$ & $26 \cdot 0$ & $24 \cdot 1,28 \cdot 0$ & $22 \cdot 9$ & $21 \cdot 5,24 \cdot 4$ & 20.5 & $18 \cdot 8,22 \cdot 4$ \\
\hline \multicolumn{9}{|l|}{ Ethnic group } \\
\hline White & $32 \cdot 9$ & $31 \cdot 8,34 \cdot 0$ & $27 \cdot 4$ & $26 \cdot 1,28 \cdot 7$ & $25 \cdot 6$ & $24 \cdot 6,26 \cdot 6$ & $24 \cdot 2$ & $22 \cdot 8,25 \cdot 7$ \\
\hline Other & $26 \cdot 5$ & $22 \cdot 8,30 \cdot 6$ & $23 \cdot 6$ & $18 \cdot 8,29 \cdot 3$ & $18 \cdot 1$ & $14 \cdot 7,21 \cdot 9$ & $15 \cdot 1$ & $12 \cdot 1,18 \cdot 8$ \\
\hline
\end{tabular}

(OR 0.69; 95\% CI 0.62, 0.77). These results were not materially changed in sensitivity analysis using logistic regression (Table 4).

\section{Discussion}

The present study indicates that in England, over the period 1997-2007, there was a steady decline in salt use at the table, and importantly that the reduction in salt use at the table was significantly greater after the introduction of the FSA's salt reduction campaign in 2004. Salt use at the table accounts for $15-20 \%$ of total salt intake, and this is the first time that the impact of the FSA salt reduction campaign on salt use at the table has been quantified. The present analysis also suggests that adding salt at the table was more common in males, those of white ethnicity, those living in the north of England and those from lower total household income groups.

Compared with other studies that have used small, single surveys ${ }^{(12,21)}$, the present study has several strengths, including the use of the large, nationally representative HSE datasets and the use of repeated cross-sectional surveys which

Table 3. Ordinal logistic regression analyses to identify independent predictors of salt behaviour ${ }^{\star}$ at the table, 2003-7 (weighted estimates) (Odds ratios and $95 \%$ confidence intervals)

\begin{tabular}{|c|c|c|c|c|c|c|}
\hline & \multicolumn{3}{|c|}{ Unadjusted } & \multicolumn{3}{|c|}{ Adjusted $\dagger$} \\
\hline & OR & $95 \% \mathrm{Cl}$ & $P$ & OR & $95 \% \mathrm{Cl}$ & $P$ \\
\hline \multicolumn{7}{|l|}{ Policy } \\
\hline Pre-policy & 1.00 & Reference & & 1.00 & Reference & \\
\hline Post-policy & 0.61 & $0.58,0.64$ & $<0.001$ & 0.58 & $0.54,0.63$ & $<0.001$ \\
\hline Age (years) & 1.00 & $1.00,1.02$ & 0.234 & 0.98 & $0.96,0.99$ & $<0.001$ \\
\hline \multicolumn{7}{|l|}{ Sex } \\
\hline Male & 1.00 & Reference & & 1.00 & Reference & \\
\hline Female & 0.76 & $0.73,0.79$ & $<0.001$ & 0.71 & $0.68,0.74$ & $<0.001$ \\
\hline \multicolumn{7}{|l|}{ Region } \\
\hline North & 1.00 & Reference & & 1.00 & Reference & \\
\hline Midlands & 0.90 & $0.83,0.98$ & 0.003 & 0.90 & $0.84,0.98$ & 0.011 \\
\hline South & 0.80 & $0.75,0.85$ & $<0.001$ & 0.82 & $0.77,0.88$ & $<0.001$ \\
\hline \multicolumn{7}{|c|}{ Total household income } \\
\hline Low & 1.00 & Reference & & 1.00 & Reference & \\
\hline Medium & 0.83 & $0.78,0.88$ & $<0.001$ & 0.79 & $0.75,0.84$ & $<0.001$ \\
\hline High & 0.79 & $0.72,0.86$ & $<0.001$ & 0.75 & $0.69,0.82$ & $<0.001$ \\
\hline \multicolumn{7}{|l|}{ Ethnicity } \\
\hline White & 1.00 & Reference & & 1.00 & Reference & \\
\hline Non-white & 0.67 & $0.60,0.75$ & $<0.001$ & 0.69 & $0.62,0.77$ & $<0.001$ \\
\hline
\end{tabular}

* Salt use was coded as an ordered categorical variable with four levels of discretionary salt use: (1) 'generally add salt to food without tasting it first', (2) 'taste the food, but then generally add salt', (3) 'taste the food, but only occasionally add salt', or (4) 'rarely, or never, add salt at the table'.

†Adjusted for age, sex, region, total household income, ethnicity and year-specific shocks. 
Table 4. Logistic regression analysis to identify independent predictors of salt behaviour ${ }^{\star}$ at the table, 2003-7 (weighted estimates) (Odds ratios and $95 \%$ confidence intervals)

\begin{tabular}{|c|c|c|c|c|c|c|}
\hline & \multicolumn{3}{|c|}{ Unadjusted } & \multicolumn{3}{|c|}{ Adjusted $†$} \\
\hline & OR & $95 \% \mathrm{Cl}$ & $P$ & OR & $95 \% \mathrm{Cl}$ & $P$ \\
\hline \multicolumn{7}{|l|}{ Policy } \\
\hline Pre-policy & 1.00 & Reference & & 1.00 & Reference & \\
\hline Post-policy & 0.69 & $0.65,0.74$ & $<0.001$ & 0.65 & $0.59,0.72$ & $<0.001$ \\
\hline Age (years) & 1.01 & $1.00,1.03$ & 0.02 & 0.98 & $0.96,1.00$ & 0.013 \\
\hline \multicolumn{7}{|l|}{ Sex } \\
\hline Male & 1.00 & Reference & & 1.00 & Reference & \\
\hline Female & 0.76 & $0.72,0.79$ & $<0.001$ & 0.67 & $0.63,0.70$ & $<0.001$ \\
\hline \multicolumn{7}{|l|}{ Region } \\
\hline North & 1.00 & Reference & & 1.00 & Reference & \\
\hline Midlands & 0.90 & $0.83,0.98$ & 0.013 & 0.83 & $0.76,0.91$ & 0.084 \\
\hline South & 0.80 & $0.75,0.85$ & $<0.001$ & 0.80 & $0.75,0.87$ & $<0.001$ \\
\hline \multicolumn{7}{|c|}{ Total annual income } \\
\hline Low & 1.00 & Reference & & 1.00 & Reference & \\
\hline Medium & 0.83 & $0.78,0.88$ & $<0.001$ & 0.76 & $0.71,0.81$ & $<0.001$ \\
\hline High & 0.79 & $0.72,0.86$ & $<0.001$ & 0.69 & $0.62,0.77$ & $<0.001$ \\
\hline \multicolumn{7}{|l|}{ Ethnicity } \\
\hline White & 1.00 & Reference & & 1.00 & Reference & \\
\hline Non-white & 0.67 & $0.60,0.75$ & $<0.001$ & 0.73 & $0.63,0.84$ & $<0.001$ \\
\hline
\end{tabular}

* Salt use was coded as a binary variable: those who 'generally add salt at the table' and those who 'do not generally add salt at the table'.

†Adjusted for age, sex, region, total household income, ethnicity and year-specific shocks.

facilitated the assessment of annual trends using consistent data collection methods across the years. Sensitivity analysis using binary rather than ordinal logistic regression did not substantially alter the present findings.

Limitations of the HSE datasets reduced the number of years that could be included for analysis and small sample sizes in specific years meant utilising broad subcategories, particularly when stratifying by ethnic group. Other study limitations included the subjective nature of self-reported salt use, a lack of validation of the HSE methods to monitor dietary behaviour $^{(22)}$ and the unavailability of sample weights before 2003, which reduced the number of pre-campaign years included in the present analysis. The possible influence of unobserved variables on salt behaviour might also lead to uncertainty in attributing the reduction in salt use to the FSA's campaign. However, the inclusion of dummy variables (one for each year) in our model allowed us to estimate the change in salt use associated with the policy over and above trends due to unobserved influences, such as the apparent decrease in salt use at the table from 1997.

The present results are consistent with previous findings of a beneficial effect of the salt campaign on self-reported consumer behaviour ${ }^{(10,11,21,23)}$, and add to this evidence base by identifying actual changes in behaviour (salt use at the table) across different sociodemographic profiles. The present results confirm previous findings that highlight greater use of salt by males $^{(12,24)}$, those in lower socio-economic groups ${ }^{(12,24-27)}$ and those living in the north of England ${ }^{(9)}$.

While the present analysis suggests a step change in salt use at the table after the introduction of the salt campaign in 2003, the observed trends (Fig. 1) suggest that the proportion of adults in England adding salt at the table remained fairly stable after 2005. Furthermore, in 2007, there remained approximately a quarter of all adults in England who add salt at the table, suggesting that continued efforts are needed in order to maintain the achieved salt reductions in the UK population.

The observed demographic differences in salt use indicate a need for future efforts to be tailored to specific target groups. It is particularly important for public health interventions that require behaviour change to be effective in reaching socially disadvantaged populations to avoid increasing already present disparities between socio-economic groups. Although there is some evidence to suggest that this is possible ${ }^{(26)}$, a lack of research into the effectiveness of health promotion techniques in lower socio-economic status groups hampers the progress in the reduction of health inequalities ${ }^{(26)}$. The prevalence of CVD increases with age and is higher in men than in women ${ }^{(28)}$, suggesting that future salt reduction strategies might usefully target men who currently report greater salt use at the table. The lower use of salt at the table in nonwhite ethnic groups may reflect previous findings of high salt use during cooking in these groups ${ }^{(24,29)}$. With high rates of hypertension and previous research indicating a lack of awareness of government guidance on salt use in South Asian and African populations ${ }^{(24,29)}$, future campaign efforts should also focus on limiting salt use in these populations ${ }^{(30)}$. Subsequent phases of the salt campaign, 2005, 2007 and 2009, warrant further analysis once more rounds of HSE data are available, and future campaigns would benefit from the incorporation of specific and rigorous impact evaluations.

Changing consumer behaviour forms only part of a national strategy to reduce population salt intake, and other strategies such as the reduction of salt in processed foods are critical and have already shown considerable successes ${ }^{(31,32)}$. Recent commitments by the food industry under the Department of Health's 'Public Health Responsibility Deal' in England to further reduce salt in processed foods are welcomed. 
However, reliance on industry reformulation may underestimate the potential impact of a health education strategy, and public awareness campaigns, together with regular evaluations, have been recommended as a central component to a successful population-wide salt reduction strategy ${ }^{(31,33)}$. Salt intake levels in the UK remain well above target levels of $6 \mathrm{~g} / \mathrm{d}$ and further efforts are still needed. Future programmatic and research efforts may benefit from focusing on those population groups such as men, and poorer families, whose current salt intake and health profile are the most worrying.

\section{Acknowledgements}

We gratefully acknowledge the Economic and Social Data Service for granting permission to access and use the HSE datasets. The original data creators, depositors or copyright holders, the funders of the HSE Data Collections and the UK Data Archive bear no responsibility for their further analysis or interpretation. A. D. D. and B. S. identified the study topic and design. J. S. obtained the survey data, conducted the statistical analysis and wrote the first draft. P. E. provided the statistical support. All authors contributed to and approved the final draft of the paper. This study received no specific grant from any funding agency in the public, commercial or not-for-profit sectors. All authors declare that there are no conflicts of interest.

\section{References}

1. World Health Organization (2003) Diet, Nutrition and the Prevention of Chronic Diseases: Report of a Joint WHO/ FAO Expert Consultation. Geneva: WHO.

2. Department of Health (1994) Nutritional Aspects of Cardiovascular Disease. Report on Health and Social Subjects 46. London: HMSO.

3. Scientific Advisory Committee on Nutrition (2003) Salt and Health. London: The Stationery Office.

4. He FJ \& MacGregor GA (2009) A comprehensive review on salt and health and current experience of worldwide salt reduction programmes. J Hum Hypertens 23, 363-384.

5. Henderson L, Irving K, Gregory J, et al. (2003) The National Diet and Nutrition Survey: Adults Aged 19 to 64 Years. London: The Stationery Office.

6. Food Standards Agency (2007) Campaign continues to drive down salt consumption and improve public health. http:// www.food.gov.uk/news/pressreleases/2007/mar/saltconsumptioncampaign?view=printerfriendly (accessed 15 July 2011).

7. Food Standards Agency (2008) Dietary sodium levels surveys. http://tna.europarchive.org/20110116113217/tna. europarchive.org/20110116113217/http://www.food.gov. uk/science/dietarysurveys/urinary (accessed 15 July 2011).

8. Sadler K, Nicholson S, Steer T, et al (2011) National diet and nutrition survey - assessment of dietary sodium in adults (aged 19 to 64 years) in England, 2011. https://www.wp.dh. gov.uk/transparency/files/2012/06/Sodium-Survey-England2011_Text_to-DH_FINAL1.pdf (accessed 1 July 2011).

9. Shankar B, Brambila-Macias J, Traill B, et al. (2012) An evaluation of the UK Food Standards Agency's salt campaign. Health Econ (Epublication ahead of print version 6 January 2012).

10. Food Standards Agency (2005) Putting the Consumer First: Developmental Report Spring 2005. London: HMSO.
11. Food \& Health Alliance (2009) Food Standards Agency salt campaign: measuring success. http://www.fhascot.org.uk/ Resource/food-standards-agency-salt-campaign-measuringsuccess (accessed 1 July 2011).

12. Jenner K (2010) Salt and your health: TNS Public Opinion Survey. http://www.actiononsalt.org.uk/Docs/33386. pdf (accessed 23 July 2011).

13. Joint Health Surveys Unit of Social and Community Planning Research and University College London. Health Survey for England, 1997 [computer file]. 3rd ed. Colchester, Essex: UK Data Archive [distributor], SN: 3979. http://dx.doi.org/ 10.5255/UKDA-SN-3979-1

14. National Centre for Social Research and University College London, Department of Epidemiology and Public Health. Health Survey for England, 1998 [computer file]. 5th ed. Colchester, Essex.: UK Data Archive [distributor], SN: 4150. http://dx.doi.org/10.5255/UKDA-SN-4150-1

15. National Centre for Social Research and University College London, Department of Epidemiology and Public Health. Health Survey for England, 2003 [computer file]. 2nd ed. Colchester, Essex: UK Data Archive [distributor], SN: 5098. http://dx.doi.org/10.5255/UKDA-SN-5098-1

16. National Centre for Social Research and University College London, Department of Epidemiology and Public Health. Health Survey for England, 2005 [computer file]. 3rd ed. Colchester, Essex: UK Data Archive [distributor], SN: 5675. http://dx.doi.org/10.5255/UKDA-SN-5675-1

17. National Centre for Social Research and University College London, Department of Epidemiology and Public Health. Health Survey for England, 2006 [computer file]. 4th ed. Colchester, Essex: UK Data Archive [distributor], SN: 5809. http://dx.doi.org/10.5255/UKDA-SN-5809-1

18. National Centre for Social Research and University College London. Department of Epidemiology and Public Health. Health Survey for England, 2007 [computer file]. 2nd ed. Colchester, Essex: UK Data Archive [distributor], SN: 6112. http://dx.doi.org/10.5255/UKDA-SN-6112-1

19. Crockett A (updated by Afkhami R, Rafferty A, Higgins V, Marshall A) (2011) Weighting the Social Surveys. London: ESDS Government.

20. Morris S, Sutton M \& Gravelle H (2005) Inequity and inequality in the use of health care in England: an empirical investigation. Soc Sci Med 60, 1251-1266.

21. Food Standards Agency (2011) Biannual Public Attitudes Tracker Survey. http://www.food.gov.uk/science/socsci/ surveys/publictrackingsurvey (accessed 32 July 2011).

22. Roberts K (2010) Dietary Surveillance and Nutritional Assessment in England: What is Measured and Where are the Gaps? Oxford: National Obesity Observatory.

23. Wyness LA, Butriss JL \& Stanner SA (2012) Reducing the population's sodium intake: the UK Food Standards Agency's salt reduction programme. Pub Health Nutr 15, 254-261.

24. Millett C, Laverty AA, Stylianou N, et al. (2012) Impacts of a national strategy to reduce population salt intake in England: serial cross sectional study. Plos One 7, e29836.

25. Darmon N \& Drewnowski A (2008) Does social class predict diet quality? Am J Clin Nutr 87, 1107-1117.

26. Michie S, Jochelson K, Markham WA, et al. (2009) Lowincome groups and behaviour change interventions: a review of intervention content, effectiveness and theoretical frameworks. J Epidemiol Commun Health 63, 610-622.

27. Purdy J, Armstrong G \& McIlveen H (2002) The influence of socio-economic status on salt consumption in Northern Ireland. Int J Consum Stud 26, 71-80. 
28. Scarborough P, Bhatnagar P, Wickramasinghe K, et al. (2010) Coronary Heart Disease Statistics. London: Department of Public Health, University of Oxford.

29. Masango M \& Kohckhar S (2009) Domestic use of salt shows high salt consumption in Black Africans and Indian Asians associated with a very low awareness level of national salt guidelines. Proc Nutr Soc 68, E153.

30. Scarborough P, Bhatnagar P, Kaur A, et al. (2010) Ethnic Differences in Cardiovascular Disease. London: Department of Public Health, University of Oxford.
31. Nutbeam D (2000) Health literacy as a public health goal: a challenge for contemporary health education and communication strategies into the 21st century. Health Promot Int 15, 259-267.

32. He FJ \& MacGregor GA (2010) Reducing population salt intake worldwide: from evidence to implementation. Prog Cardiovasc Dis 52, 363-382.

33. Cappuccio FP, Capewell S, Lincoln P, et al. (2011) Policy options to reduce population salt intake. $\mathrm{Br} \mathrm{Med} \mathrm{J}$ 343, d4995. 